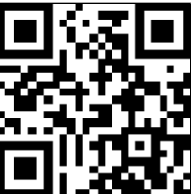

Editor's choice

to access free content

Centre for Sports and Exercise Medicine, Queen Mary University of London, London, UK

\section{Correspondence to}

Dr Dylan Morrissey, Centre for Sports and Exercise Medicine, Queen Mary University of London Mile End Hospital, Bancroft road, London E1 4DG, UK;

d.morrissey@qmul.ac.uk

Received 11 January 2012

Revised 26 July 2012

Accepted 2 August 2012

Published Online First

3 September 2012

\title{
Gluteal muscle activity and patellofemoral pain syndrome: a systematic review
}

\author{
Christian J Barton, Simon Lack, Peter Malliaras, Dylan Morrissey
}

ABSTRACT

Objective There is growing evidence to support the association of gluteal muscle strength deficits in individuals with patellofemoral pain syndrome (PFPS) and the effectiveness of gluteal strengthening when treating PFPS. In additiona, an impressive body of work evaluating gluteal electromyography (EMG) has recently emerged, further supporting the importance of gluteal muscle function in PFPS. This systematic review synthesises these EMG findings in order to better understand the role of gluteal muscle activity in the aetiology, presentation and management of PFPS. Methods MEDLINE, EMBASE, CINAHL, Web of Knowledge and Google Scholar databases were searched in September 2011 for prospective and case-control studies evaluating the association of gluteal EMG with PFPS. Two independent reviewers assessed each paper for inclusion and quality. Means and SDs were extracted from each included study to allow effect size calculations and comparison of results.

Results Ten case-control, but no prospective studies were identified. Moderate-to-strong evidence indicates gluteus medius (GMed) activity is delayed and of shorter duration during stair negotiation in PFPS sufferers. In addition, limited evidence indicates GMed activity is delayed and of shorter duration during running, and gluteus maximus (GMax) activity is increased during stair descent.

Conclusions Delayed and shorter duration of GMed EMG may indicate impaired ability to control frontal and transverse plane hip motion. Further research evaluating the value of gluteal muscle activity screening in identifying individuals most likely to develop PFPS, and the effectiveness of interventions targeting changes to gluteal muscle activation patterns is needed.

\section{INTRODUCTION}

Patellofemoral pain syndrome (PFPS) is one of the most common presentations to sports medicine practitioners. In a large study of 2519 presentations to a sports medicine clinic, $5.4 \%$ were diagnosed with PFPS, accounting for $25 \%$ of all knee injury presentations. ${ }^{1}$ In additiona, incidence estimates range between $9 \%$ and $15 \%$ in active populations such as athletes and military recruits. ${ }^{2-10}$ Put together, these statistics highlight the importance of understanding aetiology and developing effective management strategies for PFPS.

Despite debate regarding the source of pain, ${ }^{11}$ consensus that PFPS results due to altered or elevated lateral patellofemoral joint (PFJ) stress currently exists. ${ }^{1-14}$ Multiple factors are thought to lead to altered lateral PFJ stress, with various extrinsic and intrinsic biomechanical characteristics thought to be involved. One particular intrinsic biomechanical factor that has received increasing attention within previous literature is neuromuscular control at the knee and the hip. Traditionally, research has focused on muscle function of the vastii, with imbalance between vastus medialis oblique (VMO) and vastus lateralis (VL) thought to elevate lateral PFJ stress. ${ }^{15}$ Providing tentative evidence to support this theory, a recent systematic review and meta-analysis reported a delayed onset of $\mathrm{VMO}$ relative to $\mathrm{VL}$ may exist in some individuals with PFPS. ${ }^{16}$ In additional, previous research indicates reversal of this delay through physiotherapy may be associated with better clinical outcomes. ${ }^{17}$

With developing clarity about the role of muscles acting primarily at the knee, it is an ideal time to consider neuromuscular control of the hip in more detail. Recent research and theoretical analyses ${ }^{18}$ have expanded the neuromuscular control focus to address this. It is theorised that impaired gluteal muscle function may result in increased hip joint adduction and internal rotation movement during activities such as running, squatting and stair negotiation. This excessive hip motion is proposed to increase lateral PFJ stress, associated with PFPS development. ${ }^{18}$ Supporting this theory, gluteal muscle strengthening programmes have been associated with positive clinical outcomes. ${ }^{19}{ }^{20}$ In additional, a recent systematic review ${ }^{21}$ found that individuals with PFPS exhibit reduced gluteus medius (GMed) and gluteus maximus (GMax) muscle strength.

Despite the growing evidence to support the efficacy of gluteal muscle strengthening ${ }^{19} 20$ and indicating individuals with PFPS possess impaired gluteal muscle strength, ${ }^{21}$ one recent prospective study reported PFPS development may not be predicted through gluteal muscle strength testing, ${ }^{22}$ while another reported greater hip external rotation strength may be predictive. ${ }^{2}$ This may indicate gluteal muscle weakness develops due to the presence of PFPS rather than being an aetiological factor. ${ }^{22}$ Regardless of the true relationship, evaluating gluteal muscle strength in isolation does not provide a complete picture of the influence of gluteal muscle function on PFPS. Indeed, isometric strength tests may relate only loosely to functional muscle activity, kinematics or joint forces. Addressing this gap, an impressive body of work has recently emerged, utilising electromyography (EMG) measurement of the gluteal muscles during a range of functional tasks, often reporting differences in onset times, amplitude levels and/or activity durations between symptomatic and control participants. A systematic literature review designed to synthesise recent EMG findings in order to 
Figure 1 Flow diagram summarising study selection for inclusion.

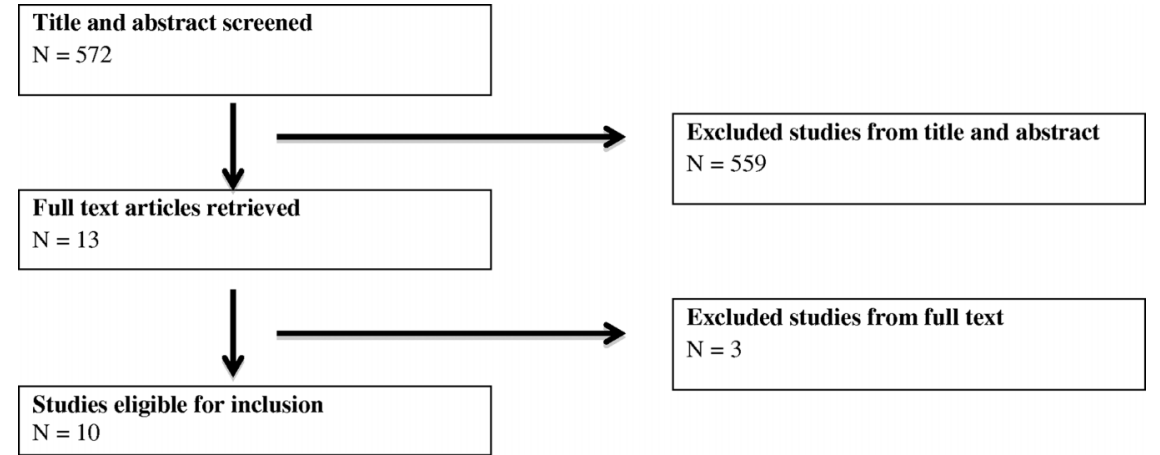

better understand the role of gluteal muscle activity in the aetiology, presentation and management of PFPS was therefore undertaken. This review aims to provide clinicians with a better understanding of the relationship between gluteal muscle activity and PFPS with the ultimate objective of facilitating improved patient management, while also identifying priorities for future research.

\section{METHODS}

\section{Inclusion and exclusion criteria}

Prospective and case-control studies evaluating gluteal EMG variables were considered for inclusion. The inclusion criteria required participants to be described as having patellofemoral pain, anterior knee pain or chondromalacia patellae. Studies including participants with other knee conditions such as patellar tendinopathy or osteoarthritis, where individuals with PFPS could not be separately analysed, were excluded.

\section{Search strategy}

MEDLINE, EMBASE, CINAHL, Web of Knowledge and Google Scholar databases were searched from inception until September 2011. A search strategy from the Cochrane systematic review on exercise therapy for PFPS was used for diagnosis search terms. ${ }^{23}$ This was then combined with the key terms EMG or muscle; and gluteal or hip or trunk or proximal. Reference lists and citing articles of included papers were also screened and a cited reference search for each included paper was completed in Google Scholar for additional publications of interest. Unpublished research was not sought. Although this may potentially lead to publication bias, ${ }^{24}$ it was deemed impractical to identify all unpublished work on EMG activity associated with PFPS from all authors and institutions around the world interested in this research area.

\section{Review process}

Titles and abstracts identified in the search were downloaded into Endnote V.X4 (Thomson, Reuters, Carlsbad, California, USA), cross referenced and any duplicates deleted. All potential publications were assessed by two independent reviewers (CB and SL) for inclusion, with full texts obtained if necessary. Any discrepancies were resolved during a consensus meeting, and a third reviewer was available if needed, but was not required.

\section{Study analysis}

Two separate scales were used to evaluate methodological quality, including a modified version of the Downs and Black Quality Index ${ }^{25}$ and the PFPS diagnosis checklist. ${ }^{26}$ Each scale was applied by two reviewers (CB and DM), with discrepancies resolved during a consensus meeting. A third reviewer was available if needed, but was not required. The diagnosis checklist is a seven-item scale summarising the reporting of key inclusion and exclusion criteria for the diagnosis of PFPS, with higher scores indicating a greater number of desired criteria had been reported. The modified version of the Downs and Black Quality Index ${ }^{25}$ is scored out of 16 , with higher scores indicating higher-quality studies. Studies with scores of 10 or greater were considered to be 'high quality' (HQ) and studies with scores below 10 were considered to be 'low quality' (LQ).

Sample sizes, participant demographics, population sources, activities, muscles and variables evaluated were also extracted.

Table 1 Study details including sample sizes, participant demographics and population sources

\begin{tabular}{|c|c|c|c|c|c|c|c|c|}
\hline \multirow[b]{2}{*}{ Paper } & \multicolumn{2}{|c|}{ Sample size } & \multicolumn{2}{|c|}{ Gender (F:M) } & \multicolumn{2}{|c|}{ Age range (mean age) } & \multicolumn{2}{|c|}{ Height (m), weight (kg) } \\
\hline & PFPS & $\overline{\mathrm{CON}}$ & PFPS & $\overline{C O N}$ & PFPS & CON & PFPS & CON \\
\hline Aminaka et $a l^{36}$ & 20 & 20 & $13: 7$ & $13: 7$ & NR $(21 \pm 4)$ & NR $(21 \pm 4)$ & $1.70 \pm 0.10,71 \pm 15$ & $1.72 \pm 0.09,70 \pm 10$ \\
\hline Brindle et $a l^{39}$ & 16 & 12 & $12: 4$ & $7: 5$ & $18-35$ (NR) & $18-35$ & $N R, N R$ & $N R, N R$ \\
\hline Boling et $a l^{34}$ & 14 & 14 & $9: 5$ & $9: 5$ & $18-42(24 \pm 6)$ & $18-42(23 \pm 2)$ & $1.68 \pm 0.10,72 \pm 12$ & $1.71 \pm 0.07,72 \pm 16$ \\
\hline Cowan et $a l^{32}$ & 10 & 27 & $7: 3$ & $15: 12$ & $18-40(26 \pm 10)$ & $18-40(25 \pm 6)$ & $1.72 \pm 0.04,63 \pm 8$ & $1.90 \pm 0.09,69 \pm 9$ \\
\hline Earl et $a l^{33}$ & 16 & 16 & $13: 3$ & $13: 3$ & $N R(22 \pm 4)$ & NR $(21 \pm 6)$ & $1.65 \pm 0.10,62 \pm 13$ & $1.66 \pm 0.12,65 \pm 14$ \\
\hline Nakagawa et $a l^{38}$ & 9 & 10 & $9: 0$ & $10: 0$ & $18-35(23 \pm 5)$ & $18-35(23 \pm 2)$ & $1.65 \pm 0.07,61 \pm 10$ & $1.63 \pm 0.06,56 \pm 4$ \\
\hline Ott et al/ & $20 *$ & 20 & NR & NR & $18-45(21 \pm N R)$ & $18-45(23 \pm 4)$ & $1.71 \pm 0.07,70 \pm 8$ & $1.68 \pm 0.07,77 \pm 7$ \\
\hline Saad et $a l^{37}$ & 15 & 15 & NR & NR & NR $(23 \pm 2)$ & $N R(23 \pm 2)$ & $1.60 \pm 3,59 \pm 4$ & $1.60 \pm 3,53 \pm 2$ \\
\hline Souza and Powers ${ }^{35}$ & 21 & 20 & $21: 0$ & $20: 0$ & $18-45(27 \pm 6)$ & $18-45(26 \pm 5)$ & $1.70 \pm 0.06,65 \pm 10$ & $1.70 \pm 0.05,63 \pm 7$ \\
\hline Willson et $a l^{31}$ & 20 & 20 & $20: 0$ & $20: 0$ & $18-35(21 \pm 3)$ & $18-35(22 \pm 5)$ & $1.68 \pm 0.06,63 \pm 8$ & $1.69 \pm 0.09,62 \pm 9$ \\
\hline
\end{tabular}


Table 2 Population sources, activities, muscles and variables evaluated in each included study

\begin{tabular}{|c|c|c|c|c|}
\hline Paper & Population source & Functional activity & $\begin{array}{l}\text { Muscles } \\
\text { (method) }\end{array}$ & EMG variable \\
\hline $\begin{array}{l}\text { Aminaka } \\
\text { et } a /^{36}\end{array}$ & $\begin{array}{l}\text { University student population } \\
\text { and surrounding community }\end{array}$ & $\begin{array}{l}\text { Stair }(18 \mathrm{~cm}) \text { ascent and descent at } \\
\text { slef-selected speed }\end{array}$ & GMed (surface) & $\begin{array}{l}\text { Onset timing (threshold of } 3 \text { SD increase in EMG activity } \\
\text { from resting) } \\
\text { Duration of activity }\end{array}$ \\
\hline Brindle et $a l^{39}$ & $\begin{array}{l}\text { University student population } \\
\text { and surrounding community }\end{array}$ & $\begin{array}{l}\text { Stair }(18 \mathrm{~cm}) \text { ascent and descent at } \\
\text { natural speed }\end{array}$ & GMed (surface) & $\begin{array}{l}\text { Onset timing (threshold of } 5 \text { SD increase in EMG activity } \\
\text { from resting) } \\
\text { Duration of activity } \\
\text { Average magnitude of activity (\%MVC-linear envelope } \\
\text { from onset to offset) }\end{array}$ \\
\hline Boling et $a l^{34}$ & $\begin{array}{l}\text { One university clinic and } \\
\text { university population }\end{array}$ & $\begin{array}{l}\text { Stair }(20 \mathrm{~cm}) \text { ascent and descent at } 96 \\
\text { steps/min }\end{array}$ & GMed (surface) & $\begin{array}{l}\text { Onset timing (threshold of } 3 \text { SD increase in EMG activity } \\
\text { from resting) } \\
\text { Duration of activity }\end{array}$ \\
\hline Cowan et $a l^{32}$ & Not reported & $\begin{array}{l}\text { Stair }(22 \mathrm{~cm} \text { ) ascent (single step up as } \\
\text { quickly as possible in response to visual } \\
\text { prompt) }\end{array}$ & $\begin{array}{l}\text { Ant. GMed } \\
\text { (surface) } \\
\text { Post. GMed } \\
\text { (intramuscular) }\end{array}$ & $\begin{array}{l}\text { Onset (indentified visually) } \\
\text { Peak magnitude (\%MVC) }\end{array}$ \\
\hline Earl et $a l^{33}$ & $\begin{array}{l}\text { Three local physical therapy } \\
\text { and sports medicine clinics }\end{array}$ & $\begin{array}{l}\text { Lateral step down }(20.3 \mathrm{~cm}) \text { with a } 1 \mathrm{~s} \\
\text { descent }\end{array}$ & GMed (surface) & $\begin{array}{l}\text { Onset timing (threshold of } 3 \text { SD increase in EMG activity } \\
\text { from resting) }\end{array}$ \\
\hline \multirow[t]{2}{*}{$\begin{array}{l}\text { Nakagawa } \\
\text { et } a \beta^{38}\end{array}$} & Single physical therapy clinic & Stair $(20 \mathrm{~cm})$ descent at 96 steps/min & GMed (surface) & $\begin{array}{l}\text { Onset timing (threshold of } 2 \text { SD increase in EMG activity } \\
\text { from resting) }\end{array}$ \\
\hline & & Single leg jump as high as possible & & $\begin{array}{l}\text { Average magnitude of activity (\%MVC-linear envelope } \\
\text { from onset to foot strike) }\end{array}$ \\
\hline Ott et $a l^{40}$ & Not reported & Single leg anterior reaching task & GMed (surface) & $\begin{array}{l}\text { Average magnitude of activity (normalised to quiet } \\
\text { unilateral stance-average over period from } 500 \text { ms prior } \\
\text { to heel strike until heel strike) }\end{array}$ \\
\hline Saad et $a l^{37}$ & Not reported & $\begin{array}{l}\text { Stair }(20 \mathrm{~cm}) \text { ascent and descent at } \\
\text { natural speed }\end{array}$ & GMed (surface) & $\begin{array}{l}\text { Average magnitude of activity (linear envelope for the } \\
\text { duration of activity) }\end{array}$ \\
\hline $\begin{array}{l}\text { Souza and } \\
\text { Powers }\end{array}$ & $\begin{array}{l}\text { Convenience, local physical } \\
\text { therapy and orthopaedic } \\
\text { clinics }\end{array}$ & $\begin{array}{l}\text { Over ground running ( } 15 \mathrm{~m} \text { run-way) at } \\
3 \mathrm{~m} / \mathrm{s} \\
\text { Stair ( } 10 \% \text { body height) descent-step } \\
\text { down over a } 2 \mathrm{~s} \text { period } \\
\text { Double leg drop jump landing }(35 \mathrm{~cm})\end{array}$ & $\begin{array}{l}\text { GMed (surface) } \\
\text { GMax (surface) }\end{array}$ & $\begin{array}{l}\text { Average magnitude of activity (\%MVC - average over } \\
\text { stance period) }\end{array}$ \\
\hline Willson et $a l^{31}$ & $\begin{array}{l}\text { Three area universities and } \\
\text { two community fitness } \\
\text { centres }\end{array}$ & $\begin{array}{l}\text { Over ground running ( } 20 \mathrm{~m} \text { run-way) at } \\
\text { between } 3.52 \text { and } 3.89 \mathrm{~m} / \mathrm{s}\end{array}$ & $\begin{array}{l}\text { GMed (surface) } \\
\text { GMax (surface) }\end{array}$ & $\begin{array}{l}\text { Onset timing (threshold of } 5 \text { SD increase in EMG activity } \\
\text { from resting) } \\
\text { Duration of activity } \\
\text { Peak magnitude (\%MVC) } \\
\text { Average magnitude of activity (\%MVC-linear envelope } \\
\text { from onset to offset) }\end{array}$ \\
\hline
\end{tabular}

EMG, electromyography; GMax, gluteus maximus; GMed, gluteus medius; MVC, maximal voluntary contraction.

Means and SDs of each variable were extracted or sought from original authors to allow effect size (ES) calculations. Data were pooled where studies evaluated the same EMG variable and functional activity. Calculated individual or pooled ES were categorised as small $(\leq 0.59)$, medium $(0.60-1.19)$ or large $(\geq 1.20)$. The level of statistical heterogeneity for pooled data was established using the $\chi^{2}$ and $\mathrm{I}^{2}$ statistics (heterogeneity defined as $\mathrm{p}<0.05$ ). Definitions for 'levels of evidence' were guided by recommendations made by van Tulder $e t$ al ${ }^{27}$

Strong evidence $=$ pooled results derived from three or more studies, including a minimum of two HQ studies, which are statistically homogenous ( $p>0.05$ ) - may be associated with a statistically significant or non-significant pooled result.

Moderate evidence $=$ statistically significant pooled results derived from multiple studies, including at least one HQ study, which are statistically heterogeneous $(\mathrm{p}<0.05)$; or from multiple LQ studies which are statistically homogenous ( $\mathrm{p}>0.05$ ).

Limited evidence $=$ results from multiple LQ studies which are statistically heterogeneous $(\mathrm{p}<0.05)$; or from one HQ study.

Very limited evidence $=$ results from one LQ study.

Conflicting evidence $=$ pooled results insignificant and derived from multiple studies regardless of quality which are statistically heterogeneous $(\mathrm{p}<0.05$, ie, inconsistent).

\section{RESULTS}

Details of the search results and process of inclusion/exclusion is shown in figure 1 . Following screening of titles and abstracts, 13 publications were retained to view full text. Of these, three were excluded. Reasons for exclusion were evaluation of a nonfunctional task, ${ }^{28}$ evaluation of an elderly population ${ }^{29}$ and use of a single case study design. ${ }^{30}$ Ten case-control studies were included for final review. No prospective studies were identified. All 10 studies evaluated EMG activity of GMed, while 2 studies evaluated GMax. Study details including sample sizes and participant demographics and population sources are shown in table 1. The majority of studies contained low participant numbers, averaging just 16 PFPS and 18 control participants and only 1 completed a sample size calculation. ${ }^{31}$ Population sources, activities, muscles and variables evaluated are shown in table 2.

\section{Quality assessment}

Results from the Downs and Black scale and diagnosis checklist are shown in tables 3 and 4, respectively. Scores for the Downs and Black scale ranged from 5 to 14 of a possible 16. Of the 10 studies, 6 were rated as high-quality scoring between 11 and $14,{ }^{31-36}$ and 4 were rated as low-quality scoring between 5 and $9 .^{37-40}$ Of particular note was that only one study ${ }^{32}$ blinded 
the outcome assessor and only two studies ${ }^{31} 32$ reported the validity and reliability of their methodology. Additionally, in all lower-quality studies ${ }^{37-40}$ there was a lack of, or inadequate consideration in relation to confounding factors (items 5 and 25), and inappropriate matching between cases and controls. ${ }^{37-40}$ Scores from the diagnosis checklist ranged from 1 to 7 , indicating large heterogeneity in reporting and/or definition of inclusion/exclusion criteria used.

\section{Onset time of muscle activation}

Seven studies ${ }^{31-34} 363839$ evaluated GMed onset timing during functional tasks, and one study ${ }^{31}$ evaluated GMax (see figure 2). Strong evidence indicates individuals with PFPS exhibit delayed GMed onset during stair descent (two $\mathrm{HQ}^{34} 36$ and two $\mathrm{LQ}^{38} 39$ studies; $\left.\mathrm{I}^{2}=51 \%, \mathrm{p}=0.10\right)$, with a small pooled $\mathrm{ES}$ $(-0.53,-0.91$ to -0.15$)$. Moderate evidence indicates that individuals with PFPS exhibit delayed GMed onset during stair ascent (three $\mathrm{HQ}^{32} \quad 34 \quad 36$ and one $\mathrm{LQ}^{39}$ study; $\mathrm{I}^{2}=67 \%$, $\mathrm{p}=0.02)$ with a small ES $(-0.52,-0.85$ to -0.19$)$. Limited evidence indicates individuals with PFPS exhibit delayed GMed onset during running (one $\mathrm{HQ}$ study ${ }^{31}$ ) with a medium ES $(-0.74,-1.38$ to -0.10$)$. Single HQ studies indicate limited evidence that GMed timing is not different during a lateral step down, ${ }^{33}$ and GMax timing is not different during running. ${ }^{31}$ In addition, one LQ study indicates very limited evidence that GMed timing is not different during a single leg jumping task. ${ }^{38}$

\section{Duration of muscle activation}

Four studies 31343639 evaluated duration of muscle activity for GMed during functional tasks, and one study ${ }^{31}$ evaluated GMax (see figure 3). Strong evidence indicated individuals with PFPS demonstrate a shorter duration of GMed activity during stair ascent (two $\mathrm{HQ}^{34} 36$ and one $\mathrm{LQ}^{39}$ study; $\mathrm{I}^{2}=32 \%$, $\mathrm{p}=0.23)$, with a small pooled ES $(-0.43,-0.84$ to -0.02$)$. Moderate evidence indicates individuals that with PFPS exhibit a shorter duration of GMed activity during stair descent (two $\mathrm{HQ}^{34} 36$ and one $\left.\mathrm{LQ}^{39} ; \mathrm{I}^{2}=70 \%, \mathrm{p}=0.04\right)$ with a medium ES $(-0.91,-1.34$ to -0.47$)$. Limited evidence indicates individuals with PFPS exhibit a shorter duration of GMed activity during running (one HQ study ${ }^{31}$ ) with a medium ES $(-0.85,-1.50$ to -0.20). A single HQ study indicates limited evidence that GMax timing is not different during running. ${ }^{31}$

\section{Muscle activation levels}

Five studies ${ }^{31} \quad \begin{array}{lllll}35 & 37 & 38 & 40 & \text { evaluated muscle activation levels }\end{array}$ (peak or average/linear envelope) for GMed during functional tasks, and two evaluated GMax (see figure 4). Only one variable was found to significantly differ, with limited evidence indicating increased average GMax activity during stair descent (one HQ study $\left.^{35}\right)$, with a medium ES $(0.80,0.16$ to 1.44$)$. Moderate evidence indicates no differences in GMed average activity during running (two $\mathrm{HQ}^{31} 35$ studies; $\mathrm{I}^{2}=2 \%, \mathrm{p}=0.31$ ). Limited evidence from one HQ study ${ }^{31}$ indicates no difference in peak GMed or GMax during running and drop jump landing. Very limited evidence indicates no difference in average GMed activity during walking, ${ }^{38}$ stair ascent, ${ }^{37}$ a single leg vertical jump ${ }^{38}$ or single leg anterior reach task. ${ }^{40}$ Conflicting evidence was found for average GMed activity during stair descent (one HQ and two LQ studies; $\mathrm{I}^{2}=79 \%, \mathrm{p}=0.009$ ) and average GMax activity during running (two HQ; $\mathrm{I}^{2}=80 \%, \mathrm{p}=0.02$ ).

\section{DISCUSSION}

This systematic review was completed to synthesise findings from previous research evaluating the association of gluteal

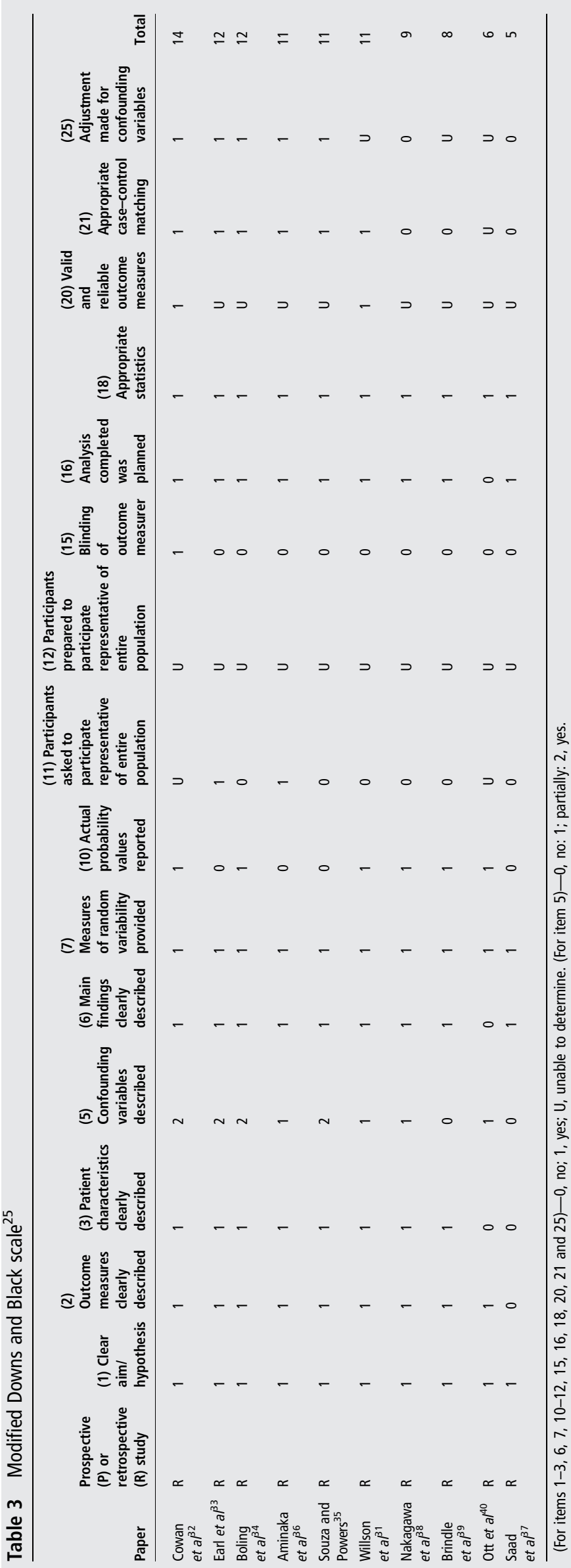


Table 4 Patellofemoral pain syndrome diagnosis checklist ${ }^{26}$

\begin{tabular}{|c|c|c|c|c|c|c|c|c|}
\hline \multirow[b]{2}{*}{ Paper } & \multicolumn{3}{|c|}{ Inclusion items } & \multicolumn{4}{|c|}{ Exclusion items } & \multirow[b]{2}{*}{$\begin{array}{l}\text { Total } \\
\text { score }\end{array}$} \\
\hline & $\begin{array}{l}\text { Clear } \\
\text { definition of } \\
\text { location }\end{array}$ & $\begin{array}{l}\text { Insidious onset } \\
\text { unrelated to } \\
\text { trauma }\end{array}$ & $\begin{array}{l}\text { Symptoms } \\
\text { consistent with } \\
\text { diagnosis }\end{array}$ & $\begin{array}{l}\text { Previous } \\
\text { knee surgery }\end{array}$ & $\begin{array}{l}\text { Internal } \\
\text { derangement }\end{array}$ & $\begin{array}{l}\text { Ligamentous } \\
\text { instability }\end{array}$ & $\begin{array}{l}\text { Other sources of } \\
\text { anterior knee } \\
\text { pain }\end{array}$ & \\
\hline Souza and Powers ${ }^{35}$ & 1 & 1 & 1 & 1 & 1 & 1 & 1 & 7 \\
\hline Cowan et $a^{\beta 2}$ & 1 & 1 & 1 & 0 & 1 & 1 & 1 & 6 \\
\hline Nakagawa et al ${ }^{38}$ & 1 & 1 & 1 & 0 & 1 & 1 & 1 & 6 \\
\hline Ott et al ${ }^{40}$ & 1 & 1 & 0 & 1 & 1 & 1 & 1 & 6 \\
\hline Aminaka et $a /^{36}$ & 1 & 1 & 1 & 1 & 0 & 0 & 1 & 5 \\
\hline Willson et $a^{\beta^{31}}$ & 1 & 1 & 1 & 0 & 1 & 1 & 0 & 5 \\
\hline Boling et $a l^{34}$ & 1 & 1 & 1 & 1 & 0 & 0 & 0 & 4 \\
\hline Brindle et $a^{39}$ & 0 & 1 & 1 & 0 & 1 & 1 & 0 & 4 \\
\hline Earl et $a \beta^{33}$ & 0 & 0 & 1 & 1 & 0 & 0 & 0 & 2 \\
\hline Saad et $a l^{37}$ & 0 & 0 & 0 & 1 & 0 & 0 & 0 & 1 \\
\hline
\end{tabular}

muscle activity with PFPS. There is currently moderate to strong evidence that GMed muscle activity is delayed and of shorter duration during stair ascent and descent in individuals with PFPS. In additiona, limited evidence indicates that GMed muscle activity is delayed and of shorter duration during running. Limited evidence indicated that GMax muscle activity was increased during stair descent. However, the remaining findings related to activation levels for both GMed and GMax were generally inconsistent, possibly owing to heterogeneity of the condition, varying methodological quality, the small number of studies in some areas and/or data reduction and processing procedures of included studies.

\section{Onset time and duration of muscle activation}

Pooled data indicated moderate-to-strong evidence for delayed, and shorter, duration of GMed during stair negotiation; and limited evidence indicated delayed and shorter duration of GMed during running in individuals with PFPS. However, these activation pattern differences were not consistent across all studies and tasks (see figures 2 and 3). Importantly, findings from Boling et $a l^{34}$ indicated a trend towards earlier GMed

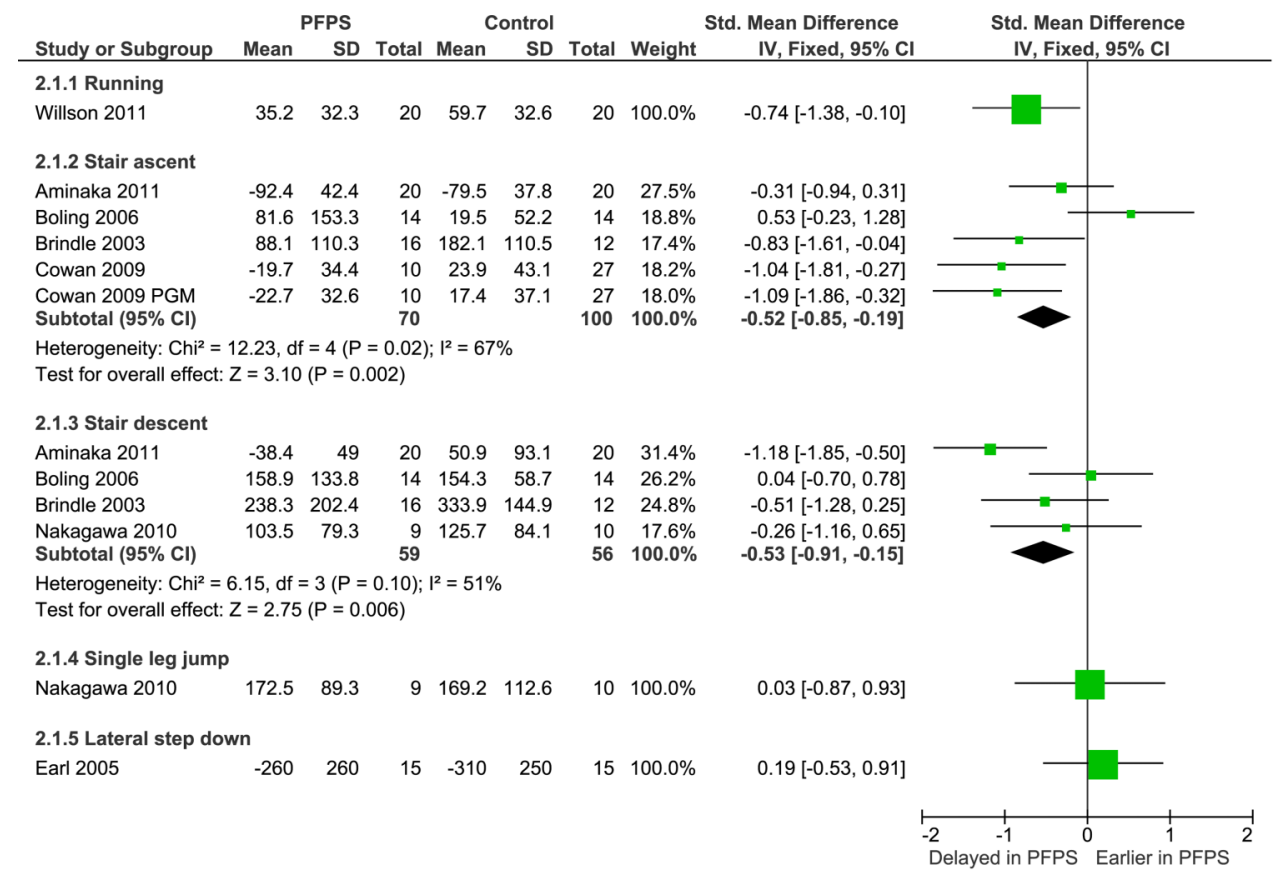

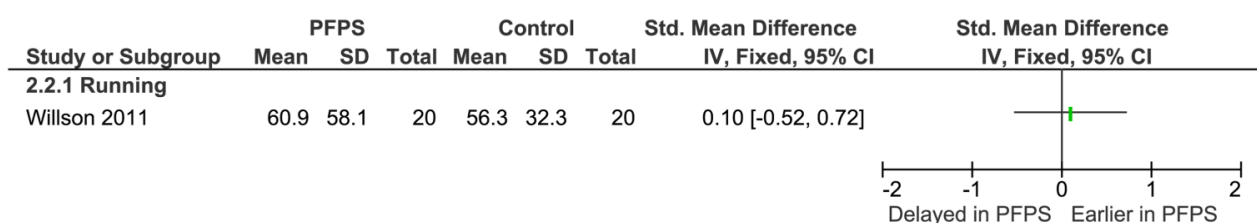

Figure 2 Gluteal electromyography onset times during various functional tasks. (A) Gluteus medius and (B) Gluteus maximus. PFPS, patellofemoral pain syndrome; PGM, posterior gluteus medius (indwelling electrode). This figure is only reproduced in colour in the online version. 


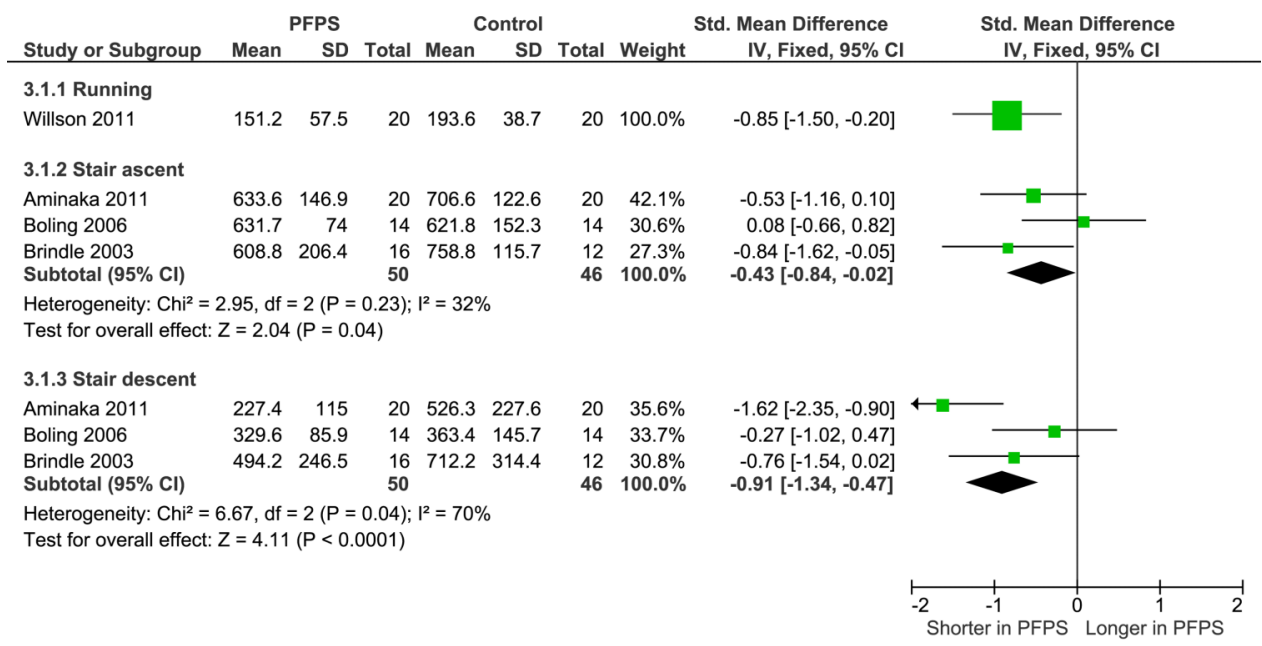

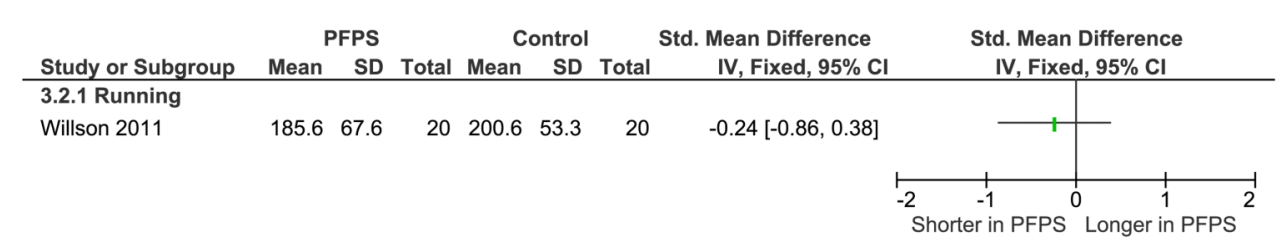

Figure 3 Gluteal electromyography durations during various functional tasks. (A) Gluteus medius and (B) Gluteus maximus. PFPS, patellofemoral pain syndrome. This figure is only reproduced in colour in the online version.

muscle activity during stair ascent (see figure 2A). When attempting to identify methodological differences to explain this disparate finding, it appears the methods for identifying GMed onset time were similar in the study by Boling et $a l^{34}$ (>3 SD above resting EMG activity) to other studies ${ }^{36} 39$ (>3-5 SD above resting EMG activity) where onsets were significantly later during stair negotiation. In additiona, the age and gender balances were also similar across the related studies ${ }^{32} 34 \quad 3639$ (see table 1) and no key consistent difference in inclusion/exclusion criteria including pain and function levels was apparent (see table 4). Therefore, this difference may reflect the multifactorial nature of PFPS.

Altered patterns of GMed muscle activity (ie, delayed onset and shorter duration) identified in this review may be a primary factor associated with PFPS in some individuals, although without prospective research it cannot be determined whether this relationship is one of cause or effect. Regardless, delayed and shorter duration of GMed may provide an explanation for greater hip adduction and internal rotation reported in some previous PFPS case-control studies evaluating lower-limb kinematics. ${ }^{41}$ If gluteal muscle activation is delayed, frontal and transverse plane hip motion control may be impaired, leading to increased stress on the PFJ and subsequent symptoms associated with PFPS. Supporting this theory, Willson $e t a l^{31}$ recently reported a moderate correlation between delayed GMed onset time and greater magnitude of hip adduction excursion during running. Further research is needed to determine the relationship of GMed onset time and duration on kinematics at the hip and ultimately PFJ loading.

\section{Muscle activation levels}

For the majority of comparisons, GMed and GMax muscle activity levels did not differ between groups (see figure 4). This may indicate that the level of gluteal muscle activation is of less importance than activation pattern in relation to pathology in PFPS. However, prospective evaluation of gluteal muscle activity in those who develop PFPS is required to confirm this.
Conflicting findings related to average GMed muscle activity during stair descent and average GMax activity during running may be explained by methodological differences between identified studies. During running, Souza et $a l^{35}$ established average GMax activity over the stance period (ie, from foot strike until toe off), with findings indicating a significant increase in activity for the PFPS group. However, Willson $e a^{31}$ established average GMax from activity onset to offset, taking into account activation prior to heel strike. Their findings indicated no differences between groups. Considering the methodological differences, these findings may indicate that the gluteal musculature demonstrates reduced activity prior to foot strike, followed by increased activity in response to loading in individuals with PFPS. Further evaluation of these different approaches and periods of activity in the same cohorts is needed in future research.

Conflicting findings were produced by three studies evaluating average GMed muscle activities during stair descent (see figure 4A). Specifically, one study ${ }^{38}$ indicated a significant increase, one study ${ }^{35}$ indicated a non-significant increase and one study ${ }^{37}$ indicated a significant decrease in activity in individuals with PFPS. One possible explanation for these conflicting findings may be the lack of control in relation to cadence by Saad et $a l,{ }^{37}$ whose findings indicated a significant decrease in average GMed activity during stair descent. Decreased ground reaction force was also found in the study by Saad et al, ${ }^{37}$ possibly indicating reduced cadence and an attempt to reduce load on the PFJ. Such a reduction in ground reaction force may ultimately reduce the work required by the gluteal musculature and subsequent EMG activity. Both Nakagawa et $a l^{38}$ and Souza and Powers ${ }^{35}$ controlled cadence that may have reduced the ability to compensate, with their findings indicating greater GMed activity during stair descent. However, without evaluation of ground reaction force (GRF) in these studies, this possibility cannot be confirmed. Further research evaluating the influence of cadence and GRF on gluteal muscle activity is needed to provide clarity. 


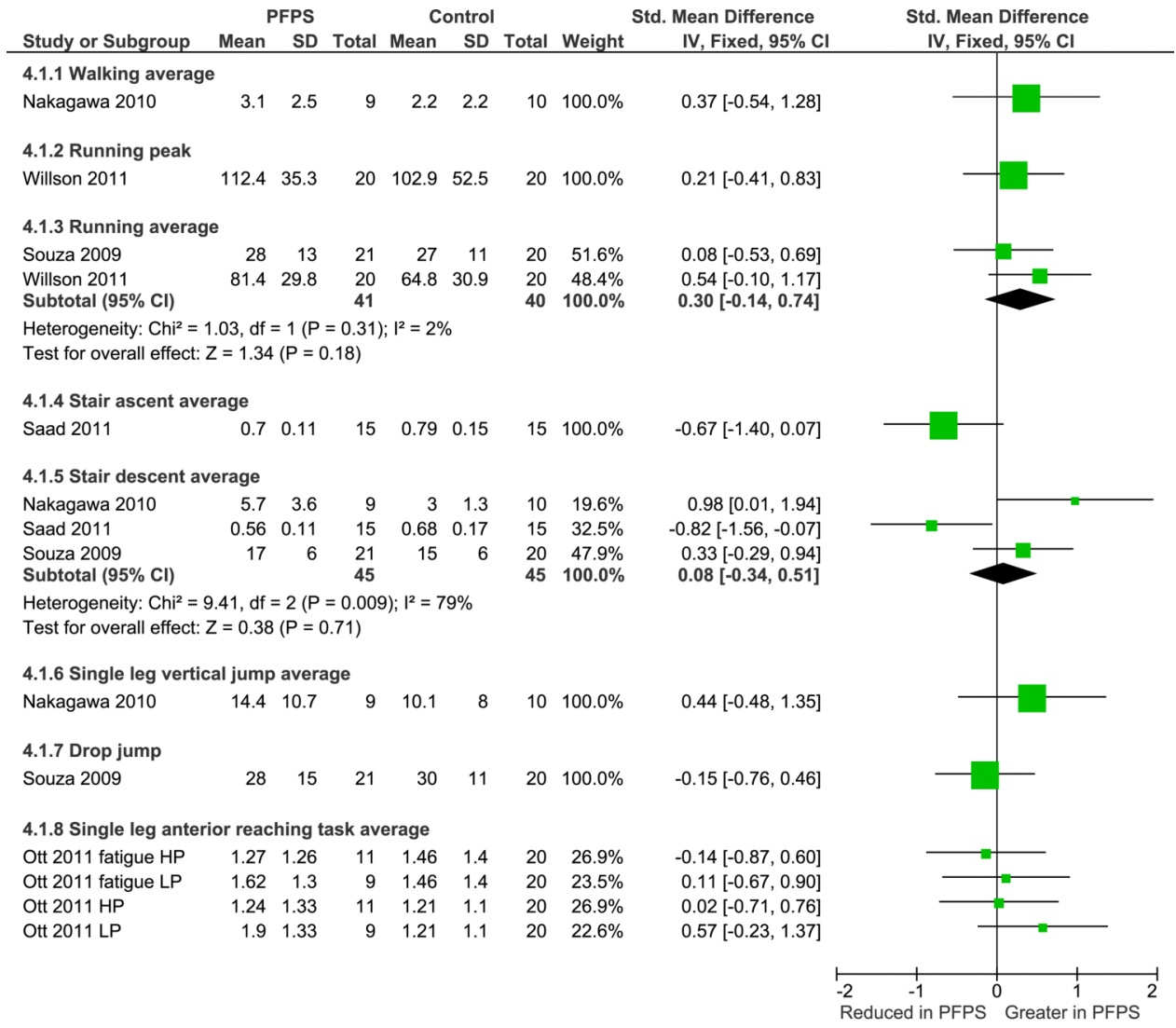

\begin{tabular}{|c|c|c|c|c|c|c|c|c|c|}
\hline \multirow[b]{2}{*}{ Study or Subgroup } & \multicolumn{3}{|c|}{ PFPS } & \multicolumn{3}{|c|}{ Control } & \multicolumn{2}{|c|}{ Std. Mean Difference } & \multirow{2}{*}{$\begin{array}{c}\text { Std. Mean Difference } \\
\text { IV, Fixed, } 95 \% \mathrm{Cl}\end{array}$} \\
\hline & Mean & SD & Total & Mean & SD & Total & Weight & IV, Fixed, $95 \% \mathrm{Cl}$ & \\
\hline \multicolumn{10}{|l|}{ 4.2.1 Running peak } \\
\hline Willson 2011 & 76.4 & 36.4 & 20 & 89.2 & 42.6 & 20 & $100.0 \%$ & $-0.32[-0.94,0.31]$ & \\
\hline \multicolumn{10}{|c|}{ 4.2.2 Running average } \\
\hline Souza 2009 & 44 & 31 & 21 & 23 & 11 & 20 & $48.2 \%$ & $0.88[0.23,1.52]$ & \\
\hline $\begin{array}{l}\text { Willson } 2011 \\
\text { Subtotal (95\% Cl) }\end{array}$ & 51.6 & 27.3 & $\begin{array}{l}20 \\
41\end{array}$ & 56.1 & 30.1 & $\begin{array}{l}20 \\
40\end{array}$ & $\begin{array}{r}51.8 \% \\
100.0 \%\end{array}$ & $\begin{array}{l}-0.15[-0.77,0.47] \\
0.34[-0.10,0.79]\end{array}$ & \\
\hline \multicolumn{10}{|c|}{$\begin{array}{l}\text { Heterogeneity: } \mathrm{Ch}^{2}=5.09, \mathrm{df}=1(P=0.02) ; l^{2}=80 \% \\
\text { Test for overall effect: } Z=1.50(P=0.13)\end{array}$} \\
\hline \multicolumn{10}{|c|}{ 4.2.3 Stair descent average } \\
\hline Souza 2009 & 15 & 9 & 21 & 9 & 5 & 20 & $100.0 \%$ & $0.80[0.16,1.44]$ & \\
\hline \multicolumn{10}{|c|}{ 4.2.4 Drop jump average } \\
\hline Souza 2009 & 30 & 10 & 21 & 32 & 10 & 20 & $100.0 \%$ & $-0.20[-0.81,0.42]$ & \\
\hline
\end{tabular}

Figure 4 Gluteal electromyography activation levels during various functional tasks. (A) Gluteus medius and (B) Gluteus maximus. PFPS, patellofemoral pain syndrome. This figure is only reproduced in colour in the online version.

\section{Clinical implications}

Findings from this systematic review indicate that delayed and shorter duration of gluteal muscle activity may exist in individuals with PFPS. Considering this, specifically targeting interventions towards correcting these deficits (eg, biofeedback or gait retraining) should also be considered in the management of PFPS. Further research evaluating the effectiveness of such strategies compared to, or combined with effective hip-strengthening programmes ${ }^{42-45}$ is needed. This systematic review has identified a large level of heterogeneity in the findings related to gluteal muscle activity characteristics associated with PFPS. Although this may reflect varying methodological design, it may also be a function of the multifactorial nature of the condition, highlighting the importance of not considering hip muscle function in isolation when treating PFPS.

\section{Methodological considerations and directions for future research}

A number of methodological limitations were identified following application of the modified Downs and Black Quality Index, ${ }^{25}$ including the absence of outcome measurer blinding and reporting of validity/reliability of methodology; lack of, or inadequate consideration in relation to confounding factors such as control of gait velocity/cadence and inappropriate matching on participant characteristics such as age, height, weight and gender between cases and controls. These areas should be addressed in future research. In additiona, many non-significant findings in this review may be the result of low participant numbers and the absence of a sample size calculation, a weakness that should be addressed in future research. 
The SENIAM guidelines ${ }^{46}$ provide clear and valid guidance regarding the preparation and application of electrodes during the collection of gluteal EMG. However, the same clear guidance is lacking for data collection procedures, reduction and analysis. As a result, these methodological aspects varied across the included studies (see table 2), possibly explaining some of the conflicting findings. Unfortunately, without direct evaluation comparing outcomes due to varied approaches in the same cohorts, it is difficult to establish the exact nature or size of their influence on results. Future studies evaluating gluteal EMG in individuals with PFPS should consider addressing this. In particular, the influence of cadence, method of identifying muscle onset time and method of establishing EMG activity levels on results needs to be established.

The ability to distinguish between cause and effect in relation to identified differences is impaired by the absence of prospective research. Additional research is needed to determine if screening of gluteal muscle activity can successfully identify those most likely to develop PFPS. Findings from case-control studies were inconsistent for all variables evaluated. This may be a function of the large heterogeneity in methodological design, and in particular inconsistent inclusion/exclusion criteria for diagnosis. It is recommended that future case-control studies use inclusion/exclusion criteria checklist ${ }^{26}$ to guide participant recruitment which is based on high-quality randomised controlled trials evaluating conservative PFPS interventions. ${ }^{47} 48$

\section{CONCLUSION}

Current research evaluating the association of gluteal muscle activity with PFPS is limited by an absence of prospective research, low sample sizes and heterogeneity in methodological design including procedures, data reduction and analysis and participant inclusion and exclusion criteria. Conflicting findings may be a function of these methodological differences and/or the multifactorial nature of PFPS. Moderate-to-strong evidence indicates that GMed muscle activity is delayed and of shorter duration during stair ascent and descent in individuals with PFPS. Additionally, limited evidence indicates that GMed muscle activity is delayed and of shorter duration during running, and GMax muscle activity is increased during stair descent. Further research evaluating the value of gluteal muscle activity screening in identifying individuals most likely to develop PFPS is needed. Additionally, evaluating the effectiveness of interventions such as biofeedback and gait retraining targeting changes of gluteal muscle activation patterns is needed.

Contributors All authors contributed significantly to the article formulation. SL and CJB had the original idea and led on the writing. PM and DM assisted this process. DM also did the quality assessment.

Competing interests None.

Provenance and peer review Not commissioned; externally peer reviewed.

\section{REFERENCES}

1 Devereaux MD, Lachmann SM. Patello-femoral arthralgia in athletes attending a Sports Injury Clinic. Br J Sports Med 1984;18:18-21.

2 Boling MC, Padua DA, Marshall SW, et al. A prospective investigation of biomechanical risk factors for patellofemoral pain syndrome: the Joint Undertaking to Monitor and Prevent ACL Injury (JUMP-ACL) cohort. Am I Sports Med 2009;37:2108-16

3 Finestone A, Radin EL, Lev B, et al. Treatment of overuse patellofemoral pain. Prospective randomized controlled clinical trial in a military setting. Clin Orthop Rel Res 1993;293:208-10.

4 Hetsroni I, Finestone A, Milgrom C, et al. A prospective biomechanical study of the association between foot pronation and the incidence of anterior knee pain among military recruits. J Bone Joint Surg, Br Volume 2006:88:905-8.
5 Milgrom C, Finestone A, Eldad A, et al. Patellofemoral pain caused by overactivity. A prospective study of risk factors in infantry recruits. J Bone Joint Surg, Am Volume 1991:73:1041-3.

6 Phillips J, Coetsee MF. Incidnce of non-traumatic anterior knee pain among 11-17-year olds. S Afr J Sports Med 2007;19:60-4.

7 Schwellnus MP, Jordaan G, Noakes TD. Prevention of common overuse injuries by the use of shock absorbing insoles. A prospective study. Am I Sports Med 1990;18:636-41.

8 Wills AK, Ramasamy A, Ewins DJ, et al. The incidence and occupational outcome of overuse anterior knee pain during army recruit training. J R Army Med Corps 2004;150264-9.

9 Witvrouw E, Lysens R, Bellemans J, et al. Intrinsic risk factors for the development of anterior knee pain in an athletic population. A two-year prospective study. Am J Sports Med 2000;28:480-9.

10 Myer GD, Ford KR, Foss KDB, et al. The incidence and potential pathomechanics of patellofemoral pain in female athletes. Clin Biomech 2010;25:700-7.

11 Davis IS, Powers CM. Patellofemoral pain syndrome: proximal, distal, and local factors, an international retreat, April 30-May 2, 2009, Fells Point, Baltimore, MD. J Orthop Sports Phys Ther 2009;40:A1-16.

12 Feller JA, Amis AA, Andrish JT, et al. Surgical biomechanics of the patellofemoral joint. Arthroscopy 2007;23:542-53.

13 Heino Brechter J, Powers CM. Patellofemoral joint stress during walking in persons with and without patellofemoral pain. Med Sci Sports Exerc 2002;34:1582-93.

14 Heino Brechter J, Powers CM. Patellofemoral joint stress during stair ascent and descent in persons with and without patellofemoral pain. Gait Posture 2002;16:115-23.

15 McConnell J. Management of patellofemoral problems. Man Ther 1996;1:60-6.

16 Chester R, Smith TO, Sweeting D, et al. The relative timing of VMO and VL in the aetiology of anterior knee pain: a systematic review and meta-analysis. BMC Musculoskelet Disord 2008;9:64.

17 Cowan SM, Bennell KL, Crossley KM, et al. Physical therapy alters recruitment of the vasti in patellofemoral pain syndrome. Med Sci Sports Exerc 2002;34:1879-85.

18 Powers CM. The influence of abnormal hip mechanics on knee injury: a biomechanical perspective. J Orthop Sports Phys Ther 2010:40:42-51.

19 Fukuda TY, Rossetto FM, Magalhaes E, et al. Short-term effects of hip abductors and lateral rotators strengthening in females with patellofemoral pain syndrome: a randomized controlled clinical trial. J Orthop Sports Phys Ther 2010;40: $736-42$.

20 Mascal CL, Landel R, Powers C. Management of patellofemoral pain targeting hip, pelvis, and trunk muscle function: 2 case reports. J Orthop Sports Phys Ther 2003:33:647-60.

21 Prins MR, van der Wurff P. Females with patellofemoral pain syndrome have weak hip muscles: a systematic review. Aust J Physiother 2009;55:9-15.

22 Thijs Y, Pattyn E, Van Tiggelen D, et al. Is hip muscle weakness a predisposing factor for patellofemoral pain in female novice runners? A prospective study. Am J Sports Med 2011;39:1877-82.

23 Heintjes E, Berger MY, Bierma-Zeinstra SM, et al. Exercise therapy for patellofemoral pain syndrome. Cochrane Database Syst Rev 2003:CD003472.

24 Maher C, Sherrington C, Elkins M, et al. Challenges for evidence-based physica therapy: accessing and interpreting high quality evidence on therapy. Phys Ther 2004;84:644-54.

25 Downs SH, Black N. The feasibility of creating a checklist for the assessment of the methodological quality both of randomised and non-randomised studies of health care interventions. J Epidemiol Community Health 1998;52:377-84.

26 Barton CJ, Munteanu SE, Menz HB, et al. The efficacy of foot orthoses in the treatment of individuals with patellofemoral pain syndrome: a systematic review. Sports Med 2010;40:377-95.

27 van Tulder M, Furlan A, Bombardier C, et al. Updated method guidelines for systematic reviews in the Cochrane collaboration back review group. Spine 2003:28:1290-9.

28 Baldon RM, Nakagawa TH, Muniz TB, et al. Eccentric hip muscle function in females with and without patellofemoral pain syndrome. J Athl Train 2009:44:490-6

29 Manetta J, Franz LH, Moon C, et al. Comparison of hip and knee muscle moments in subjects with and without knee pain. Gait Posture 2002;16:249-54.

30 Albisetti W, De Bartolomeo 0, Gabbiadini S, et al. Surface EMG evaluation of patellofemoral pain syndrome in a professional ballet dancer. Med Probl Perform Artist 2008;23:29-32.

31 Willson JD, Kernozek TW, Arndt RL, et al. Gluteal muscle activation during running in females with and without patellofemoral pain syndrome. Clin Biomech (Bristol, Avon) 2011;26:735-40.

32 Cowan SM, Crossley KM, Bennell KL. Altered hip and trunk muscle function in individuals with patellofemoral pain. Br I Sports Med 2009;43:584-8.

33 Earl J, Hertel J, Denegar C. Patterns of dynamic malalignment, muscle activation, joint motion, and patellofemoral-pain syndrome. J Sport Rehabil 2005;14:215-33.

34 Boling MC, Bolgla LA, Mattacola CG, et al. Outcomes of a weight-bearing rehabilitation program for patients diagnosed with patellofemoral pain syndrome. Arch Phys Med Rehabil 2006;87:1428-35. 
35 Souza RB, Powers CM. Differences in hip kinematics, muscle strength, and muscle activation between subjects with and without patellofemoral pain. J Orthop Sports Phys Ther 2009:39:12-19.

36 Aminaka N, Pietrosimone BG, Armstrong CW, et al. Patellofemoral pain syndrome alters neuromuscular control and kinetics during stair ambulation. J Electromyogr Kinesiol 2011;21:645-51.

37 Saad MC, Felicio LR, Masullo CdL, et al. Analysis of the center of pressure displacement, ground reaction force and muscular activity during step exercises. J Electromyogr Kinesiol 2011;21:712-18.

38 Nakagawa TH, Muniz TB, Baldon RM, et al. Electromyographic preactivation pattern of the gluteus medius during weight-bearing functional tasks in women with and without anterior knee pain. Rev Bras Fisioter 2011;15:59-65.

39 Brindle TJ, Mattacola C, McCrory J. Electromyographic changes in the gluteus medius during stair ascent and descent in subjects with anterior knee pain. Knee Surg Sports Traumatol Arthrosc 2003;11:244-51.

40 Ott B, Cosby NL, Grindstaff TL, et al. Hip and knee muscle function following aerobic exercise in individuals with patellofemoral pain syndrome. J Electromyogr Kinesiol 2011;21:631-7.

41 Barton $\mathrm{C}$, Levinger $\mathrm{P}$, Menz $\mathrm{H}$, et al. Kinematic gait characteristics associated wtih patellofemroal pain syndrome: a systematic review. Gait Posture 2009;30:405-16.
42 Dolak KL, Silkman C, McKeon JM, et al. Hip strengthening prior to functional exercises reduces pain sooner than quadriceps strengthening in females with patellofemoral pain syndrome: a randomized clinical trial. J Orthop Sports Phys Ther 2011:41:560-70.

43 Earl JE, Hoch AZ. A proximal strengthening program improves pain, function, and biomechanics in women with patellofemoral pain syndrome. Am J Sports Med 2011;39:154-63.

44 Fukuda TY, Rossetto FM, Magalhaes E, et al. Short-term effects of hip abductors and lateral rotators strengthening in females with patellofemoral pain syndrome: a randomized controlled clinical trial. J Orthop Sports Phys Ther 2010;40:736-42.

45 Nakagawa TH, Muniz TB, Baldon RdM, et al. The effect of additional strengthening of hip abductor and lateral rotator muscles in patellofemoral pain syndrome: a randomized controlled pilot study. Clin Rehabil 2008;22:1051-60.

46 SENIAM. http://www.seniam.org/, (accessed 1 August 2011).

47 Collins N, Crossley K, Beller E, et al. Foot orthoses and physiotherapy in the treatment of patellofemoral pain syndrome: randomised clinical trial. BMJ 208;337: a1735.

48 Crossley K, Bennell K, Green S, et al. Physical therapy for patellofemoral pain: a randomized, double-blinded, placebo-controlled trial. Am J Sports Med 2002;30:857-65. 